\title{
Segregation of Carbon Paste in a Smelting Electrode
}

\author{
T. BERGSTRøM
}

Elkem a/s, $R \& D$ Centre, Postboks 40 Vågsbygd, N-4602 Kristiansand S., Norway

\section{S. COWLEY}

Department of Mathematics, Imperial College, London SW7 2BZ, UK

\author{
A. C. Fowler $\dagger$
}

Mathematical Institute, Oxford University, 24-29 St Giles, Oxford OX1 3LB, UK

P. E. Seward

Department of Engineering, Oxford University, Parks Road, Oxford, UK

[Received 13 November 1987 and in revised form 7 December 1988]

\begin{abstract}
An industrial problem concerning segregation in carbon paste is described. Billets of paste, which are a mixture of coke and pitch, are fed into a steel tube in a furnace, and the tube is lowered at a rate of about $0.75 \mathrm{~m} \mathrm{day}^{-1}$. In the tube, the pitch is deformable, and the paste is essentially a very viscous two-phase particulate medium. The grain size distribution is essentially bimodal, consisting of coarse $(\sim 10 \mathrm{~mm})$ particles in a distribution of finer $(100 \mu \mathrm{m})$ particles. Segregation occurs near the walls of the tube, leading to a lower content of coarse particles; such segregation is unwanted, and so the length of this segregated region is required to be minimized. It is suggested that segregation is due to the stiffness of the coarse particulate mixture, which is less able to deform than the pitch/fines mixture. Consequently, the length of the segregated region is controlled by the coarse mixture viscosity, and an expression is derived for the segregation length.
\end{abstract}

\section{Introduction}

The PROBlem to be discussed here was presented to the Mathematical Study Groups for Industry at Oxford University by Elkem a/s R \& D Centre of Norway, during the workshop in March 1987. Further discussion took place at the corresponding workshop in Edinburgh, in March 1988. Elkem apply carbon paste in the Søderberg electrode for smelting furnaces. Typical conditions in the upper part of an electrode are shown in Fig. 1. Billets of solid paste (of diameter up to $1 \mathrm{~m}$ ) are fed into the top of a cylindrical steel tube (of diameter up to $2 \mathrm{~m}$ ). Heat is supplied by fans blowing hot air along the walls, and is also induced in the steel tube by the current supplied to the contact clamps. The billets, a mixture of coke (calcined anthracite) and pitch, become deformable as the temperature increases

t Requests for reprints to A. C. Fowler. 


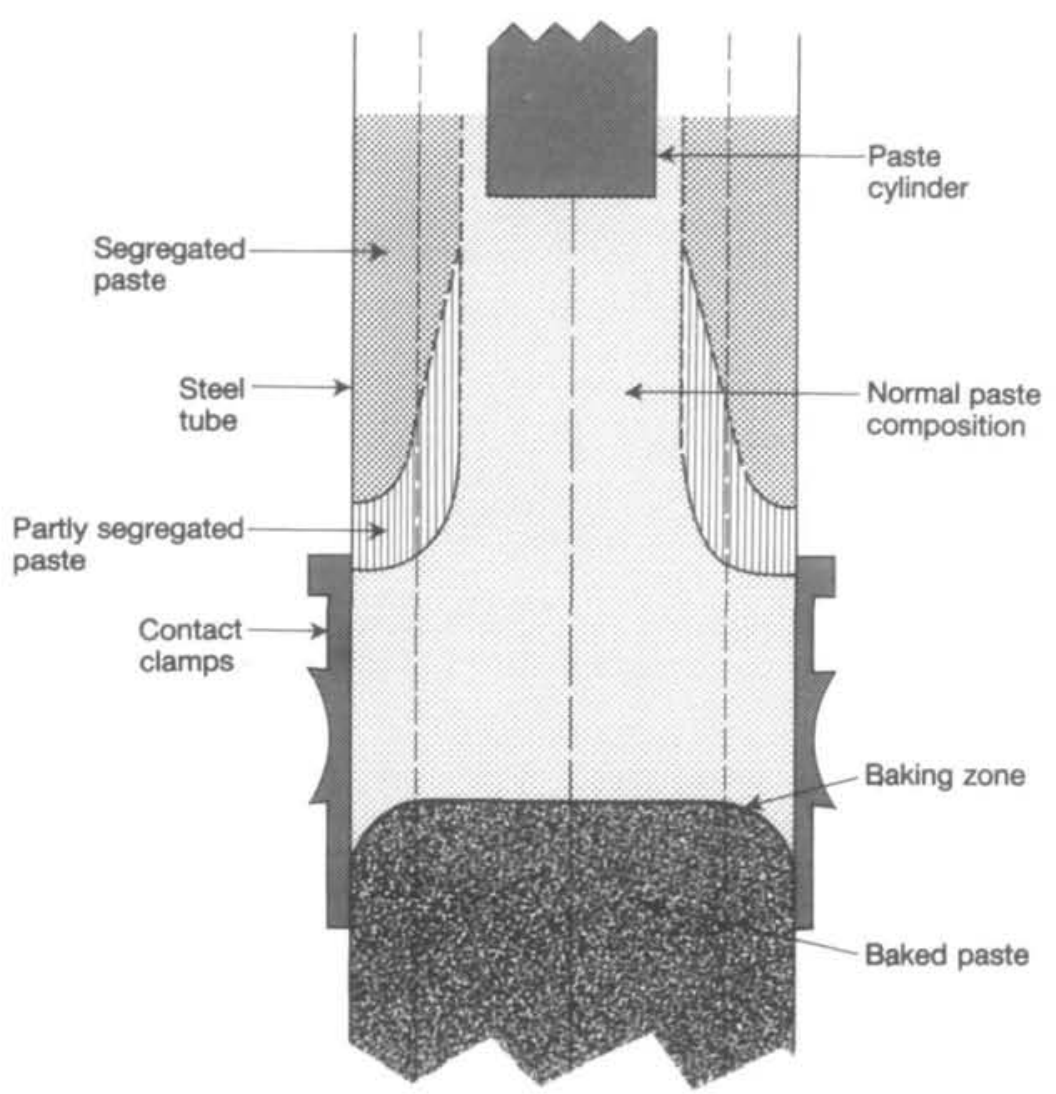

FIG. 1. A sketch of the paste in the electrode, with the segregated region indicated. This is the experimentally observed situation.

through the softening point of pitch, about $50^{\circ} \mathrm{C}$. Thus, in the cylinder, the paste is a two-phase particulate viscous medium, which can deform under the applied load (typically some $5 \mathrm{~m}$ height of billets is stacked on top). The confining tube is lowered at a rate (typically) of $0.75 \mathrm{~m} \mathrm{day}^{-1}$ (in distinct steps, though we shall consider this to be continuous), so that the flow can exist in a steady state (the input rate of billets exactly matching the consumption of baked paste at the bottom). As the paste descends, it becomes hotter, and bakes in the contact clamp area at a temperature of about $500^{\circ} \mathrm{C}$, and is thus a solid electrode conducting current to the furnace process.

A segregation zone is indicated in Fig. 1. The initial paste composition is a mixture of about $75 \%$ coke particles and $25 \%$ pitch. The distribution of coke particles (as measured) is indicated in Fig. 2a, which shows that normal paste is bimodally distributed, with a sizeable fraction (about $40 \%$ of the coke) of coarse $(10 \mathrm{~mm})$ particles, and the rest being much finer $(\sim 100 \mu \mathrm{m})$. By contrast, in the segregated region, the distribution is as shown in Fig. $2 b$ : essentially, the coarse particles are absent. 

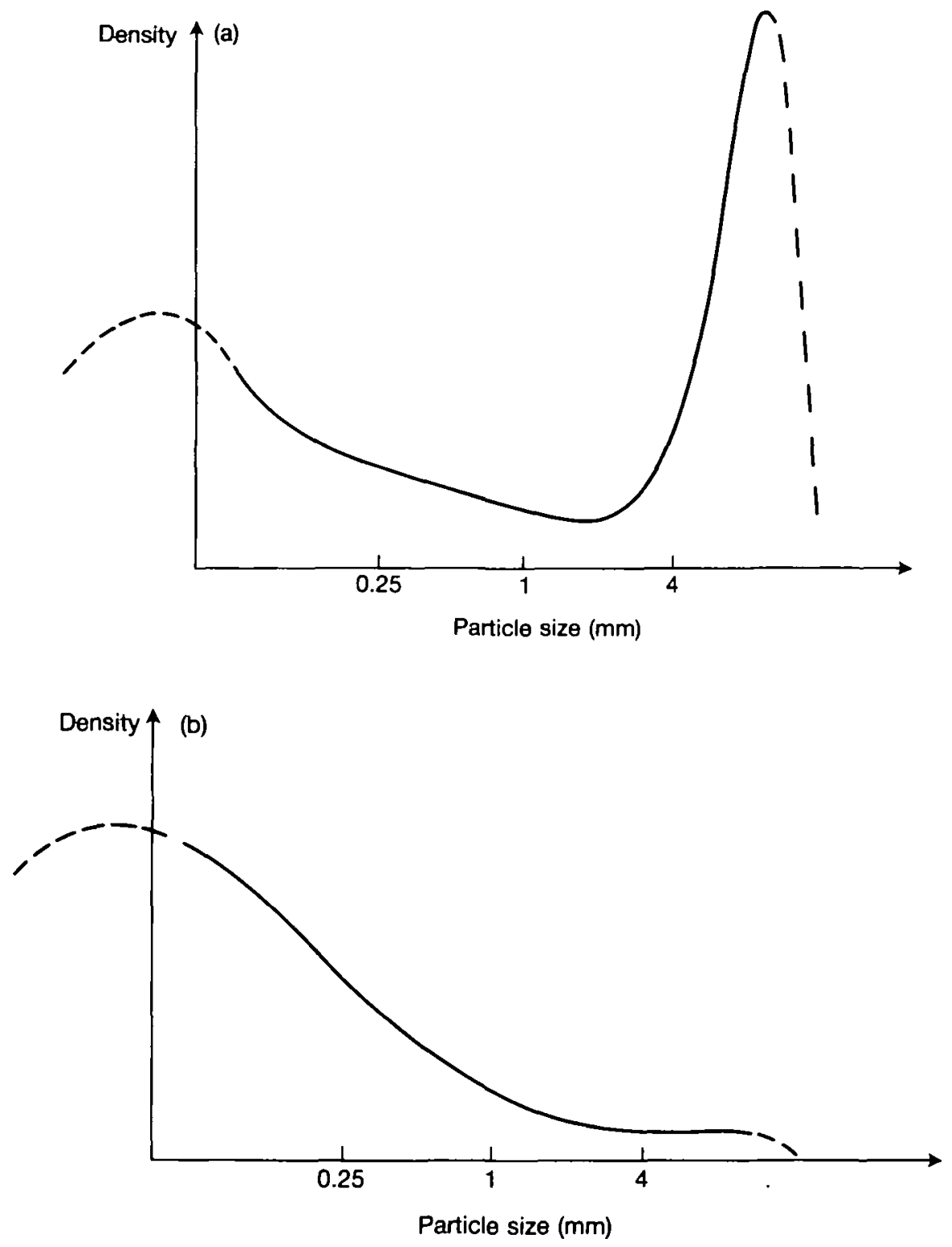

FIG. 2. Coke particle size distribution density function: (a) normal; (b) segregated. The area under the curves up to a given particle size give the total volume of coke particles less than that size. Solid lines are from measurements; dashed lines are extrapolation. Note the logarithmic scale. More specifically, if $\phi(R)$ is the fraction of particles less than radius $R$ in dimension, then these figures portray $\mathrm{d} \phi / \mathrm{d}(\ln R)$ versus $\ln R$. 

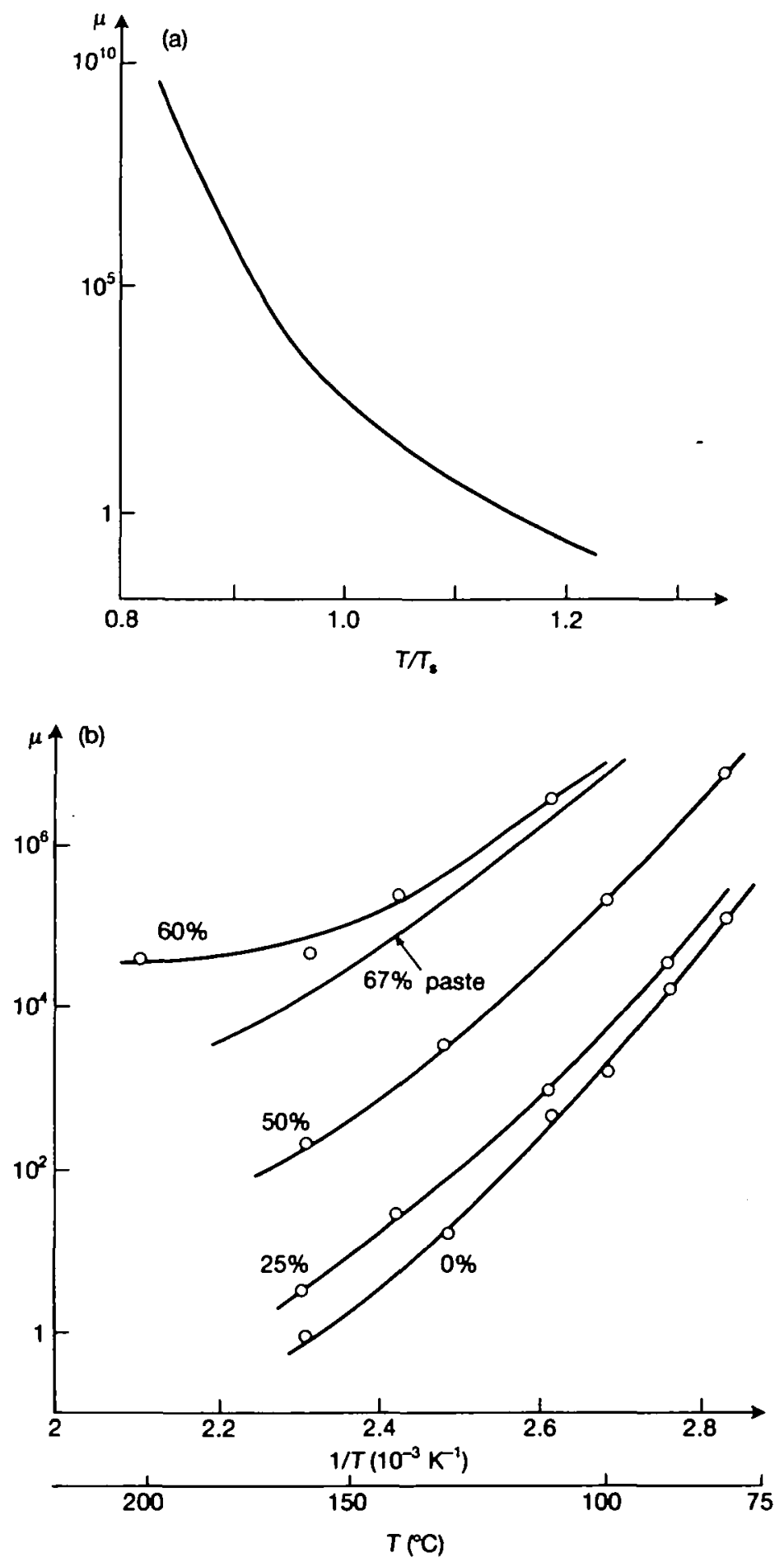

Fia. 3. Viscosity variations: (a) pure pitch, viscosity variation near softening point $T_{z} \approx 50^{\circ} \mathrm{C}$ (Sorlie, 1984); (b) pitch plus fines (sludge), for different coke fractions, versus temperature (Tørktep, 1988); also shown is the viscosity of a $67 \%$ anode paste inctuding coarse particles, but of slightly different properties; (c) sludge viscosity versus coke concentration at a fixed temperature (Sortie, unpublished data). All viscosities in $\mathbf{P a} 8$. 


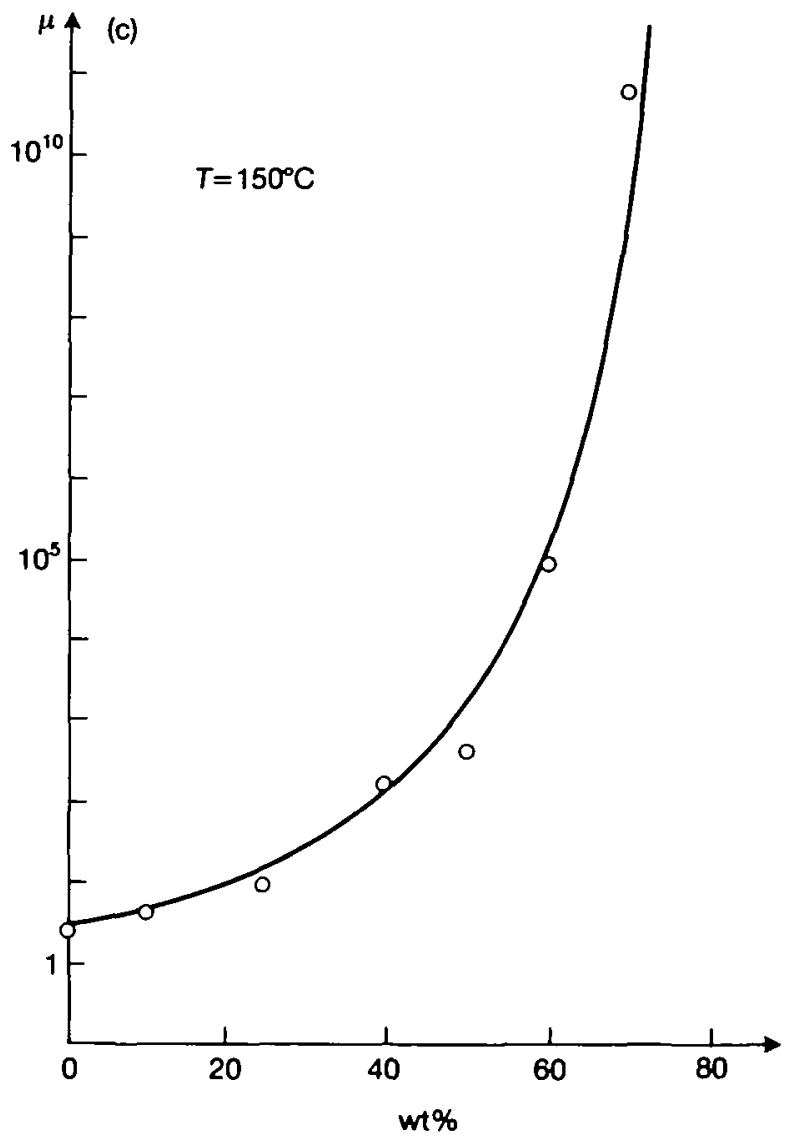

FiG. 3. (cons.)

The problem is to understand the cause of this segregation, and to quantify it. Evidently, there is relative flow involved, and it is of some interest to examine the rheology of the paste. Some data on this is shown in Fig. 3. In Fig. 3a, we see that the pitch viscosity is extremely temperature-dependent near its softening point. This corresponds to the transformation from solid billet to deformable paste. Figure $3 b$ shows some measured values of viscosity versus temperature at different concentrations of fine coke (no coarse particles). Notice that the temperature dependence of the pitch-plus-fines sludge decreases with increasing coke concentration, with a tendency at the highest concentrations to level off. Figure $3 \mathrm{~b}$ also contains data on the viscosity of a paste (pitch + fines + coarse) with $67 \%$ solids, somewhat less than the $75 \%$ quoted above. This data is for an anode paste, with rather different properties to an electrode paste, and is included in Fig. 3b to emphasize the strong temperature dependence of the mixture viscosity. It is tempting to compare this $67 \%$ paste viscosity with corresponding pitch/fines viscosities, which seem to indicate that inclusion of coarse particles makes little difference to the rheology. This is misleading for the 
following reasons: (i) the anode paste is not directly comparable to the electrode paste; (ii) the real paste has so much (75\%) coke in it that it is almost locked (see Fig. $3 c$, where it is shown that the effect of increasing concentration of fines is to increase the viscosity dramatically), so that it is reasonable to expect even $30 \%$ coarse particles to experience significantly increased resistance, compared with the fines; (iii) the simple fact that pitch plus fines does segregate from the coarse matrix suggests, ipso facto, that the rheological behaviour should be distinct. This data is taken from Tørklep (1988).

In reality, we do not expect the paste to behave like a Newtonian fluid. It is more like a saturated soil, and as such can be expected to exhibit cohesion, a yield stress, and non-Newtonian properties. Sakai $(1977 \mathrm{a}, \mathrm{b})$ has measured the viscoelastic properties of carbon paste. More suggestively, Covey \& Stanmore (1980) measured the rheological properties of wet, brown coals: they found that the behaviour could be represented as that of a (possibly nonlinear) Bingham fluid.

It is not difficult to understand how such behaviour can arise in a dense particulate paste. When there is grain-to-grain contact, then part of any external stress (e.g. overburden pressure) is taken up by intergranular contacts: this part of the stress is called the effective pressure, and will be denoted by $p_{e}$. Now, if a shear stress is applied, the paste will not deform until the stress is sufficient to overcome the internal friction due to intergranular contacts. Thus, a yield stress will exist, which we expect to depend on th 2 effective pressure. For a densely packed particulate, it is also reasonable to associate shear deformation with dilatancy - the coarse particles have to move round each other. These and other typical behaviours are discussed by Clarke (1987).

The mechanism of segregation involves the separation of the pitch/fines sludge from the coarse particulate paste. It is easy to visualize this as being due to the ability of the less viscous sludge to be squeezed out of the particulate matrix, much in the way water is squeezed out of wet sand when one stands on it. The temperature rise in the contact clamp area will (dramatically) enhance this effect. The applied load here would be the solid billets, and in this view the apparently intuitive way to reduce the segregation length would be by reducing the load. However, as we shall see, the opposite is the case. Whereas the idealization of the paste as a two-phase (sludge plus coarse particulate) medium seems appropriate, it is not so much the ease with which the sludge can be squeezed out which controls the flow, but rather the difficulty with which the coarse matrix is deformed.

The viability of this description of the paste stems from the data presented in Fig. 3. As well as the segregation of coarse from fine coke, there is also a lateral differentiation in the concentration of fine coke (possibly for similar reasons), so that the segregated sludge is about $50 \%$ fines. Although data is not available for a $75 \%$ paste, we can infer from Fig. 3b that a realistic value of paste viscosity at $150^{\circ} \mathrm{C}$ must be larger than $10^{4} \mathrm{Pas}$, possibly (by analogy with Fig. 3c) much larger, whereas at the same temperature, $50 \%$ sludge has a viscosity of $\sim 10^{2} \mathrm{~Pa}$ s. The differential viscosity implies a blocking action of the coarse particles, and hence, ipso facto, a differential flow mechanism. 


\section{Mathematical model}

We consider a 'two-phase' particulate flow. The interstitial 'liquid' is the pitch-plus-fines sludge. Suitable equations for the slow two-phase flow are then (with zero Reynolds number) (Drew, 1983)

$$
\left.\begin{array}{l}
\alpha_{t}+\nabla \cdot(\alpha w)=0, \quad-\alpha_{t}+\nabla \cdot[(1-\alpha) u]=0, \\
w-u=-K\left[\nabla p-\rho_{\ell} g l\right], \quad \nabla P=\nabla \cdot \tau+\rho_{\mathrm{m}} g l,
\end{array}\right\}
$$

where $\alpha$ is the void fraction (volume fraction of sludge), $w$ is the sludge velocity, $\boldsymbol{u}$ is the coarse particulate velocity, $p$ is the sludge pressure, and $P$ is the total pressure. We have taken the unit vector $i$ pointing vertically downwards, $g$ is the acceleration due to gravity, $\rho_{\ell}$ is the sludge density, $\rho_{m}$ is the paste density, and $\tau$ is the deviatoric stress tensor for the mixture. These equations represent conservation of mass and momentum for the two phases, and we have assumed Darcy's law for the flow of the sludge through the particulate.

Some comments on the pressures $p$ and $P$ should be made. In writing (2.1), we think of the pressure $p$ as an averaged phasic pressure (cf. Drew, 1983). That is, the total pressure $P$ would be given by $P=\alpha p+(1-\alpha) p_{m}$, where $p_{m}$ is the matrix phase-averaged pressure. However, in measuring effective and water pressures in soils, soil engineers actually measure the quantities $\alpha p$ and $(1-\alpha) p_{m}$, and it is these which they designate the interstitial pressure and effective pressure, respectively. They then determine Darcy's law experimentally in terms of the interstitial pressure. Thus, although $(2.1)_{3}$ is consistent with two-phase flow theory (Drew, 1983) if $p$ is the phasic pressure, it is conventional to think of it in measurements as being the interstitial pressure, and we shall follow this convention below. The distinction may in practice be academic (though see the discussion in Section 4), since $p$ should be related to $\alpha$ (see below).

From the above discussion, we relate $P$ and $p$ by

$$
P-p=p_{\mathrm{e}} \text {. }
$$

To complete the model, we need constitutive relations for the permeability coefficient $K$, the effective pressure $p_{e}$, and the matrix rheology (i.e. $\tau$ ). The permeability coefficient $K$ is defined by

$$
K=d^{2} / \chi \mu_{\ell},
$$

where $d$ is a typical coarse particle dimension, $\mu_{e}$ is the sludge viscosity, and $\chi$ a numerical tortuosity factor of order $10^{2}$ (Bear, 1972). In general, $K$ would vary with $\boldsymbol{\alpha}$.

Common practice in soil mechanics is to measure $p_{\mathrm{e}}$ as a function of $\alpha$ (Hillel, $1980)$ and for dynamic situations we might generalize this to

$$
p_{\mathrm{e}}=p_{\mathrm{e}}(\alpha, \nabla \boldsymbol{u}) \text {, }
$$

as has been done for boulder clays by Boulton $\&$ Hindmarsh (1987). We expect $\partial p_{e} / \partial \alpha<0$ (as in the static case). The dependence of $p_{c}$ on $\nabla u$ represents the effects of dilatancy. Thus, $\partial p_{e} / \partial\|\nabla u\|>0$ represents a dilatant material. Clarke 
(1987) suggests a similar relation (his equation (52)). A general nonlinear Bingham (or Herschel-Bulkley) fluid satisfies the flow law

$$
\dot{\varepsilon}=0 \quad\left(\tau<\tau_{c}\right), \quad \dot{\varepsilon}=A \tau^{n} p_{c}^{-q} \quad\left(\tau>\tau_{c}\right),
$$

where $\tau_{c}$ is yield stress, and $\tau$ and $\dot{\varepsilon}$ are the square roots of the second invariants of the stress and strain rate tensors (e.g. $2 \tau^{2}=\tau_{i j} \tau_{i j}$, in normal tensor notation).

It is as well to be aware of these kinds of data, but for the present purpose of practically modelling the paste flow, all we require is a flow mechanism which includes a differential viscosity between the coarse matrix and the finer sludge. Consequently, we shall henceforward suppose that a (possibly variable) viscosity $\mu_{\mathrm{m}}$ can be defined for the paste, and we shall suppose the dependence of $p_{\mathrm{e}}$ on $\alpha$ and $\|\nabla u\|$ is irrelevant; that is, we take $p_{e}$ to be constant. Notice that this may change the order or type of the equations. There is no accepted rule as to whether the strain rates in the paste flow law should refer to the coarse matrix velocity $u$, or to an averaged value $u_{m}=(1-\alpha) u+\alpha w$ (or to some other, e.g. the barycentric velocity). While the latter may seem more satisfying, we feel that the use of $u$ is more intuitively appropriate, as we visualize the coarse matrix retaining a coherent form. (Actually, for this problem, it will make no difference to the result.) It then follows that (2.1) can be written in the form

$$
\left.\begin{array}{l}
\alpha_{t}+\nabla \cdot(\alpha w)=0, \quad-\alpha_{t}+\nabla \cdot[(1-\alpha) u]=0, \\
w-u=-K\left[\nabla P-\rho_{\ell} g l\right], \quad \nabla P=\nabla \cdot\left[\mu_{m}\left\{\nabla u+(\nabla u)^{\top}\right\}\right]+\rho_{m} g i .
\end{array}\right\}
$$

These equations describe flow in the unsegregated paste. In the segregated region, coarse particles are absent, and the viscosity is much smaller. Thus the segregated region is like a pure fluid by comparison with the porous particulate matrix. Therefore, appropriate boundary conditions of continuous stress at the interface are well approximated by requiring the matrix stress to have zero shear component, and the normal stress to balance the hydrostatic pressure in the sludge. The situation is shown in Fig. 4, where we adopt cylindrical coordinates $(x, r)$ with $x$ pointing downwards. The free surface is denoted by $r=R(x, t)$. The boundary of the matrix consists of three parts on which we apply the following boundary conditions.

At the billet: $x=0,0<r<b$,

$$
\alpha=\alpha_{0}, \quad u=u_{0}, \quad v=0, \quad-\int_{\text {billet }} \sigma_{11} \mathrm{~d} S=\pi b^{2}\left(P_{0}+p_{A}\right) .
$$

Here, $(u, v)$ are longitudinal and transverse velocity components of the coarse particulate flow, $b$ is the billet radius, $\sigma_{11}$ is the (total) longitudinal stress in the $x$ direction, $p_{A}$ is atmospheric pressure, and $P_{0}$ is the applied pressure due to the billets ( $P_{0}=\rho g h$, where $h$ is the height of the billet stack).

On the free surface: $0<x<l_{3}, r=R(x, t)$, where $l_{3}$ is the segregation length,

$$
\sigma_{n t}=0, \quad \sigma_{n n}=-\left(p_{A}+\rho_{e} g x\right), \quad v=R_{t}+u R_{x} .
$$

The last relation here is the kinematic condition, which states that the free surface is a material surface for the coarse particles, which is reasonable if there is some cohesion of the matrix. 

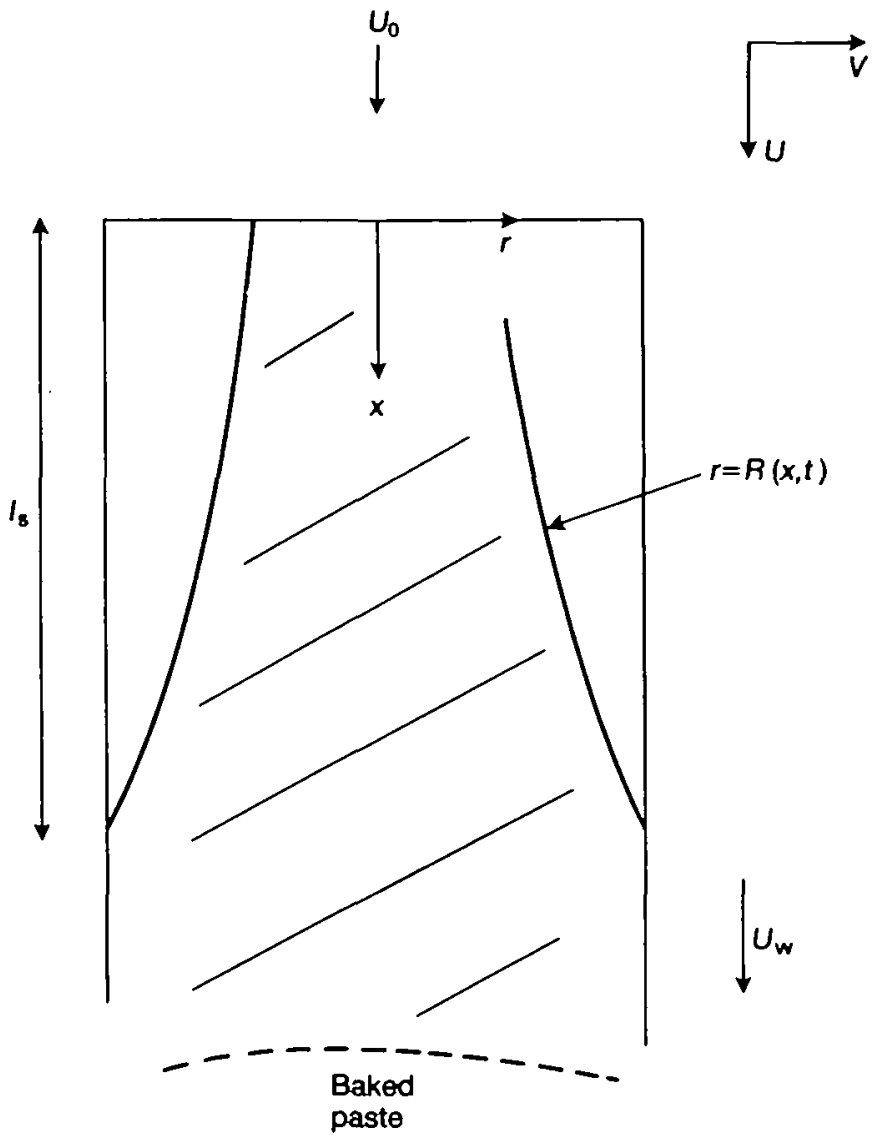

Fio. 4. Sketch of the flow configuration

On the wall: $r=a, x>l_{2}$, where $a$ is the tube radius,

$$
u=u_{w}, \quad v=0 .
$$

Here $u_{w}$ is the wall velocity, and to operate a steady state, we choose

$$
b^{2} u_{0}=a^{2} u_{w} \text {. }
$$

The above boundary conditions relate to the matrix flow. Several others (e.g. $w_{n}=0$ at the wall) would be needed for the complete problem, but we shall see that the above suffice for the present case.

\section{Analyois}

First, we reduce the equations to dimensionless form. We put

$$
\begin{aligned}
& r=a r^{*}, \quad x=a x^{*}, \quad R=a R^{*}, \quad \mu=u_{0} u^{*}, \quad w=u_{0} w^{*}, \\
& \left.t=\left(a / u_{0}\right) t^{*}, \quad P=p_{\mathrm{A}}+\rho_{\ell} g x+P_{0} P^{*}, \quad \mu_{\ell}=\mu_{\ell}^{*} \mu_{1}, \quad \mu_{\mathrm{m}}=\mu_{\mathrm{m}}^{*} \mu_{2},\right\}
\end{aligned}
$$


where the asterisks indicate dimensionless quantities. On substitution, the equations become (omitting the asterisks)

where

$$
\left.\begin{array}{ll}
\alpha_{t}+\nabla \cdot(\alpha w)=0, & -\alpha_{t}+\nabla \cdot[(1-\alpha) u]=0, \\
w-u=-\delta \mu_{1}^{-1} \nabla P, & \nabla P=\beta \nabla \cdot\left\{\mu_{2}\left[\nabla u+(\nabla u)^{\top}\right]\right\}+\gamma i,
\end{array}\right\}
$$

$$
\delta=d^{2} P_{0} / \chi \mu_{\ell}^{*} a u_{0}, \quad \beta=\mu_{\mathrm{m}}^{*} u_{0} / a P_{0}, \quad \gamma=\left(\rho_{\mathrm{m}}-\rho_{\ell}\right) g a / P_{0} .
$$

The boundary conditions (2.7)-(2.9) become

$$
\left.\begin{array}{lll}
\alpha=\alpha_{0}, \quad u=1, \quad v=0, \quad-\int_{\text {billet }} \sigma_{11} \mathrm{~d} S=\pi r_{0}^{2} & \text { at } x=0, \\
\sigma_{n t}=0, \quad \sigma_{n n}=0, \quad v=R_{t}+u R_{x} & \text { on } r=R, \\
u=r_{0}^{2}, \quad v=0 & \text { at } r=1,
\end{array}\right\}
$$

where (dimensionlessly) $\sigma=-P \delta+\beta \mu_{2}\left[\nabla u+(\nabla u)^{\top}\right], \delta$ is the unit tensor, and

$$
r_{0}=b / a \text {. }
$$

To estimate the parameters $\beta, \delta, \gamma$, we use the following rough estimates:

$$
\begin{aligned}
& a \sim 1 \mathrm{~m}, \quad u_{0} \sim 1 \mathrm{~m} \mathrm{day}^{-1} \sim 10^{-5} \mathrm{~m} \mathrm{~s}^{-1}, \quad d \sim 10 \mathrm{~mm}=10^{-2} \mathrm{~m}, \quad \chi \sim 10^{2}, \\
& \left.\begin{array}{l}
\rho_{\ell} \sim 1.6 \times 10^{3} \mathrm{~kg} \mathrm{~m}^{-3}, \quad \rho_{\mathrm{m}} \sim 1.8 \times 10^{3} \mathrm{~kg} \mathrm{~m}^{-3}, \quad g \sim 10 \mathrm{~m} \mathrm{~s}^{-2}, \quad h \sim 5 \mathrm{~m}, \\
\mu_{\mathrm{m}}^{*} \geqslant 10^{12} \mathrm{~Pa} \mathrm{~s} \text { (at } 50^{\circ} \mathrm{C}, \text { extrapolated from anode paste curve, Fig. 3b). }
\end{array}\right\}
\end{aligned}
$$

From these we compute, with $P_{0} \sim \rho g h$,

and hence

$$
P_{0} \sim 10^{5} \mathrm{~Pa},
$$

$$
\delta \sim 10^{-5}, \quad \gamma \sim 0.02, \quad \beta \geqslant 10^{2} .
$$

The inequality reflects uncertainty in $\mu_{\mathrm{m}}^{*}$. Realistically, $\delta$ could be even smaller and $\beta$ could be much larger.

Over a (segregation) length scale of order $2 \mathrm{~m}$ (see Fig. 1), the temperature rises from 50 to $\sim 150^{\circ} \mathrm{C}$, and we can expect $\mu_{1}$ and $\mu_{2}$ to decrease from $O(1)$ to $O\left(10^{-8}\right)$. We postpone such complications for the moment, and consider first the effect of the estimates in (3.8): these suggest that we neglect $\delta$ and $\gamma$ to leading order, and then adopt an expansion procedure for large $\beta$. Thus, at leading order, we have

$$
\boldsymbol{w} \sim \boldsymbol{u},
$$

whence from $(3.2)_{1,2}$ we have

and so

$$
\begin{gathered}
\nabla \cdot u=0, \\
\alpha_{\boldsymbol{t}}+u \cdot \nabla \alpha=0,
\end{gathered}
$$

$$
\alpha=\alpha_{0}
$$

in the matrix (since $\alpha=\alpha_{0}$ at $x=0$ for all time). The problem thus reduces to solving the slow flow equations

$$
\nabla \cdot u=0, \quad \nabla P=\beta \nabla \cdot\left\{\mu_{2}\left[\nabla u+(\nabla u)^{\top}\right]\right\},
$$

with the boundary conditions (3.4). 
It may be seen that the problem has an extra boundary condition due to the applied load. Nevertheless, the problem is well posed, since without this extra condition, the dimensionless segregation length

$$
c=l_{3} / a=R^{-1}(1)
$$

is arbitrary. To see this, visualize a slow viscous jet emptying from a tap into a vessel which is being lowered at a commensurate rate (so that there can be a steady state). It is physically obvious that a solution to this problem exists for any (segregation) length $c$, depending on the initial conditions. In our case, $c$ will be determined by the applied load condition.

We seek to analyse (3.13) for large $\beta$. Our ansatz is that the jet becomes long and thin, so that lubrication theory is appropriate. In cylindrical coordinates, equations (3.13) are, writing $\mu_{2}=\mu$,

$$
\left.\begin{array}{l}
u_{x}+\frac{1}{r}(r v)_{r}=0, \\
P_{x}=\beta\left(\frac{1}{r} \frac{\partial}{\partial r}\left(\mu r e_{r x}\right)+\frac{\partial}{\partial x}\left(\mu e_{x x}\right)\right), \\
P_{r}=\beta\left(\frac{1}{r} \frac{\partial}{\partial r}\left(\mu r e_{r r}\right)+\frac{\partial}{\partial x}\left(\mu e_{r x}\right)-\mu e_{\theta \theta} / r\right), \\
e_{r r}=2 \frac{\partial v}{\partial r}, \quad e_{\theta \theta}=\frac{2 v}{r}, \quad e_{x x}=2 \frac{\partial u}{\partial x}, \quad e_{r x}=\frac{\partial v}{\partial x}+\frac{\partial u}{\partial r},
\end{array}\right\}
$$

and the boundary conditions, in component form, are

$$
\begin{gathered}
u=1, \quad v=0, \quad \int_{0}^{\pi}\left(P-2 \beta \mu u_{x}\right) r \mathrm{~d} r=\frac{1}{2} r_{0}^{2} \quad \text { at } x=0, \\
R_{t}+u R_{x}=v, \quad \sigma_{1 n}=\sigma_{2 n}=0,
\end{gathered}
$$

We rescale the variables as follows:

Then (3.15) becomes

$$
x=\beta X, \quad t=\beta T, \quad v=V / \beta .
$$

$$
\left.\begin{array}{l}
u_{X}+\frac{1}{r}(r V)_{r}=0, \\
P_{X}=\frac{\beta^{2}}{r} \frac{\partial}{\partial r}\left(\mu r u_{r}\right)+\frac{1}{r} \frac{\partial}{\partial r}\left(\mu r V_{X}\right)+2 \frac{\partial}{\partial X}\left(\mu u_{X}\right), \\
P_{r}=\frac{1}{r} \frac{\partial}{\partial r}\left(2 \mu r V_{r}\right)+\frac{\partial}{\partial X}\left(\mu u_{r}\right)-2 \mu V / r^{2}+\frac{1}{\beta^{2}} \frac{\partial}{\partial X}\left(\mu V_{X}\right),
\end{array}\right\}
$$


with boundary conditions

$$
\begin{aligned}
& u=1, \quad V=0, \quad \int_{0}^{r_{0}}\left(P-2 \mu u_{X}\right) r \mathrm{~d} r=\frac{1}{2} r_{0}^{2} \quad \text { on } X=0, \\
& R_{T}+u R_{X}=V, \quad\left(P-2 \mu u_{X}\right) R_{X}+\beta^{2} \mu u_{r}+\mu V_{X}=0, \\
& -\mu\left(u_{r}+\frac{1}{\beta^{2}} V_{x}\right) R_{X}-\left(P-2 \mu V_{r}\right)=0 \quad \text { on } r=R \text {, } \\
& u=r_{0}^{2}, \quad V=0 \quad \text { on } r=1 \text {. }
\end{aligned}
$$

We seek series expansions in powers of $1 / \beta^{2}$ :

$$
u \sim u^{(0)}+\frac{1}{\beta^{2}} u^{(1)}+\cdots, \quad V \sim V^{(0)}+\cdots, \quad P \sim P^{(0)}+\cdots .
$$

(Strictly, we should expand $R$ as well, but it is not necessary here.) From these we find, successively,

$$
u^{(0)}=U(X, T)
$$

(from (3.18) $)_{2}$, and prescribing regularity at $r=0$ ), and thus from (3.18),

$$
V^{(0)}=-\frac{1}{2} r U^{\prime}, \quad U^{\prime}=U_{X} .
$$

At leading order, $(3.18)_{3}$ then yields

and $(3.18)_{2}$ leads to

$$
P^{(0)}=\tilde{P}(X, T)-\mu U^{\prime},
$$

$$
\mu u_{r}^{(1)}=\frac{1}{2} r \tilde{P}^{\prime}+\frac{1}{2} \mu r u^{\prime \prime}-\frac{3}{r} \int_{0}^{r} r \frac{\partial}{\partial X}\left(\mu U^{\prime}\right) \mathrm{d} r .
$$

Therefore, at leading order, the stress conditions on $r=R$ give

$$
\left(\tilde{P}-3 \mu U^{\prime}\right) R_{X}+\mu\left(u_{r}^{(1)}+V_{X}^{(0)}\right)=0, \quad \tilde{P}-\mu U^{\prime}-2 V_{r}^{(0)}=0,
$$

where $\mu$ is evaluated at $r=R$; these imply, using (3.22),

$$
\tilde{P}=0 \text {, }
$$

and hence from (3.25) $)_{1}$, using (3.24) and (3.22),

$$
\frac{\partial}{\partial X}\left(U^{\prime} \int_{0}^{R} r \mu \mathrm{d} r\right)=0 .
$$

Integrating (3.27), together with the applied load condition

$$
-3 U^{\prime} \int_{0}^{r_{0}} r \mu \mathrm{d} r=\frac{1}{2} r_{0}^{2} \text { when } R=r_{0}
$$

we find (with constant $P_{0}$ )

$$
U^{\prime} \int_{0}^{R} r \mu \mathrm{d} r=-\frac{1}{6} r_{0}^{2}
$$

More generally, the right-hand side may be a function of time proportional to the load. The boundary condition for (3.29) is

$$
U=1 \text { on } X=0 \text {. }
$$


In addition, application of the kinematic boundary condition on $r=R$ yields, using (3.22),

$$
R_{T}+U R_{X}=-\frac{1}{2} R U^{\prime}
$$

with boundary condition

$$
R=r_{0} \quad \text { when } x=0,
$$

and an appropriate initial condition. The segregation length $l_{\mathrm{s}}$ is now determined from $x=\beta X=l_{z} / a$ when $R=1$, that is,

$$
R\left(l_{s} / a \beta, T\right)=1 .
$$

Transient solutions to these equations can be found numerically. However, the steady-state solution is easily reduced further. Conservation of mass gives, with $R_{T}=0$,

$$
R^{2} U=\text { constant }=r_{0}^{2},
$$

and thus $U^{\prime}=-2 r_{0}^{2} R^{\prime} / R^{3}$, whence

$$
\left(\int_{0}^{R} r \mu \mathrm{d} r\right) R^{\prime} / R^{3}=\frac{1}{12}
$$

Defining $\bar{\mu}=\left(2 / R^{2}\right) \int_{0}^{R} r \mu \mathrm{d} r$ to be the cross-sectionally averaged viscosity, we find

$$
\int_{0}^{X} \mathrm{~d} X / \bar{\mu}=6 \ln R / r_{0},
$$

so that (3.33) gives the segregation length as

$$
\int_{0}^{l / a \beta} \mathrm{d} X / \bar{\mu}=6 \ln 1 / r_{0},
$$

using $X=x / \beta$. To be specific, suppose that temperature is roughly proportional to $x$, so that

and hence

$$
\bar{\mu}=\mathrm{e}^{-\lambda x}=\mathrm{e}^{-\Lambda x}, \quad \Lambda=\lambda \beta,
$$

$$
l_{\mathrm{s}}=\frac{a}{\lambda} \ln \left(1+6 \lambda \beta \ln 1 / r_{0}\right)=\frac{a \beta}{\Lambda} \ln \left(1+6 \Lambda \ln 1 / r_{0}\right) .
$$

For constant viscosity, $l_{s} \sim a \beta$, whereas, when $\Lambda \gg 1$, this is reduced to $l_{\mathrm{s}} \sim(a \beta \ln \Lambda) / \Lambda=(a \ln \beta \lambda) / \lambda$. Our previous estimates suggest that $\lambda \sim 10$ and $\Lambda \sim 10^{3}$, and one might suspect that such high values of $\Lambda$ render the lubrication approximation invalid at least in the region where the paste rapidly thickens and attaches to the cylinder wall. However, the nonuniformity in this region is likely to make only a small correction to the attachment length given by (3.39), and we do not pursue this matter further here. In any event, the lubrication approximation breaks down near the attachment point, where the jet boundary conditions change from no stress to no slip: however, we do not expect the leading order result for $l$, to alter.

If $\bar{\mu}$ is constant, we see that $R$ is an exponential (rather as shown in Fig. 4), whereas, if $\bar{\mu}$ is rapidly varying, then $R$ is almost constant until the critical 
segregation length, when the paste spreads out rapidly. This is consistent with Fig. 1. In fact, we can do rather better than (3.39) since, if $\bar{\mu}$ is rapidly varying (as it is observed to be), then a direct application of Laplace's method to (3.36) gives

$$
R \sim r_{0} \exp [-1 / 6 \beta(\mathrm{d} \bar{\mu} / \mathrm{d} x)]=r_{0} \exp [-1 / 6(\mathrm{~d} \bar{\mu} / \mathrm{d} X)],
$$

and thus $l_{\mathbf{a}}$ is determined from the condition

$$
\frac{\mathrm{d} \bar{\mu}}{\mathrm{d} x}=-\frac{1}{6 \beta \ln 1 / r_{0}}, \quad \frac{\mathrm{d} \bar{\mu}}{\mathrm{d} X}=-\frac{1}{6 \ln 1 / r_{0}} ;
$$

(i.e. $l_{2} / a$ is the value of $x$ where this relation first applies). Furthermore, data (Fig. 5) indicate that the temperature $T$ in a cross-section is coolest on the inside for small $x$, but coolest on the outside nearer the baking zone. (This is because the electrode is not at the centre of the furnace; it does not affect our analysis.) Roughly, $\bar{\mu}$ is given by the viscosity at the coolest point of the cross-section, which is thus in the interior for points above the contact clamps. The rapid rise in

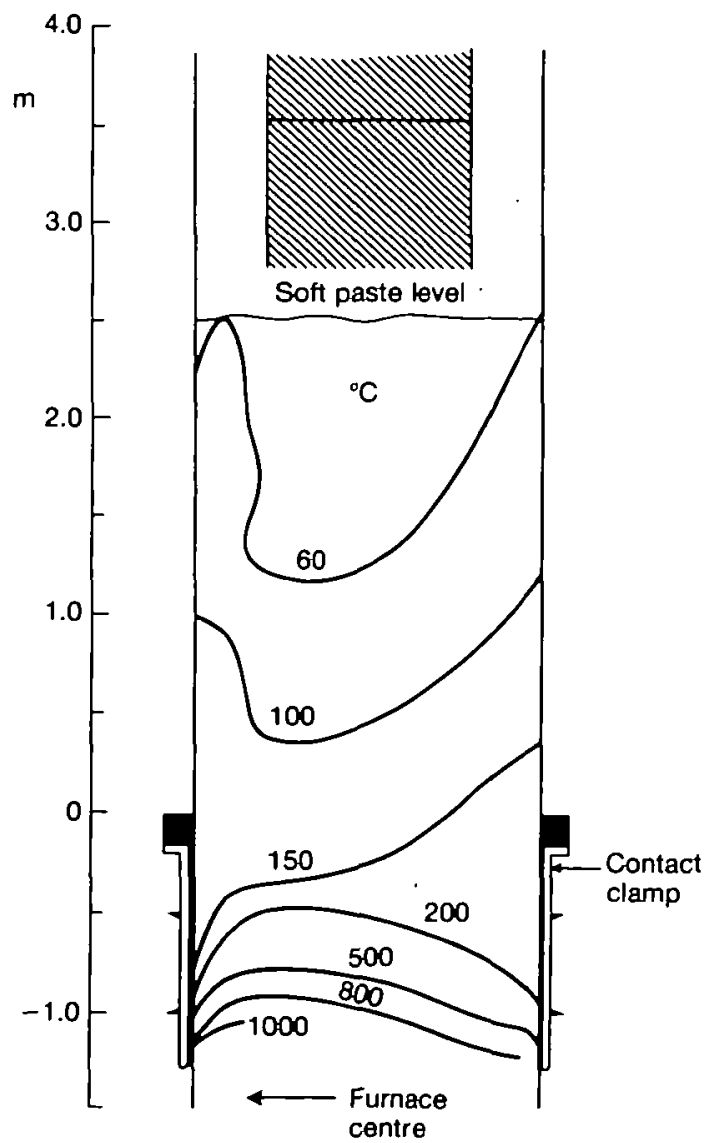

Fig. 5. Measured temperatures inside a $1.7 \mathrm{~m}$ diameter Soderberg electrode. (The temperature is not axisymmetric because the electrode is situated away from the centre of the fumace.) 
temperature gradient near the clamps is consistent with the termination there of the segregation zone. One can also treat non-Newtonian viscosity; for example, a material with viscosity function

$$
\mu=A(T) B(\dot{\varepsilon}),
$$

where $\dot{\varepsilon}$ is the second strain rate invariant, leads, as before, to (3.35). In the present approximation, $\dot{\varepsilon} \propto\left|U^{\prime}\right|$, so we can again derive (3.36) in the form

$$
\bar{A} b\left(R^{\prime} / R^{3}\right) R^{\prime} / R=\frac{1}{6},
$$

where $b \propto B$. For example, a power law such as $(2.5)_{2}$ (with $p_{\mathrm{e}}$ constant) leads to

$$
b \propto\left(R^{\prime} / R^{3}\right)^{-(n-1) / n}
$$

and $R$ can be found by quadrature.

\section{Discussion}

The specific conclusion of this analysis is that segregation occurs because of the inability of the extremely viscous matrix to deform from the incoming billets. The rate of segregation is given by (3.2) 3 , which determines the differential velocity $w-u$. In a steady state, assuming $\mu_{1}=\mu_{2}=\mu,(3.23)$ and (3.26) imply, with the rescalings in (3.17),

$$
w-u \sim-\delta \mu^{-1}\left(P_{X} / \beta, P_{r}\right) \sim \delta\left[\left(\mu u_{X}\right)_{X} / \beta \mu,-\left(\mu u_{X}\right)_{r} / \mu\right] .
$$

The dominant component (if $\mu$ varies laterally) is $w_{2} \sim \delta\left(\mu_{r} / \mu\right)\left|u_{X}\right|$. This tends to drive a weak inflow when the walls are hot, and a weak outflow when they are cold, but the effect is marginal. The point is, however, that while $(3.2)_{3}$ is responsible for segregation, it may be nevertheless insignificant when the steady segregated state has been set up. Thus, the real mechanism of segregation occurs as the viscous jet relaxes to its steady state.

A proper understanding of this requires a numerical solution, and, in passing, we mention that the problem of the numerical solution of slow viscous jet extrusion raises issues involving die swell (Middleman \& Gavis, 1961; Batchelor et al., 1973; Tanner et al., 1985) and contact line dynamics (Davis, 1983). These problems have not arisen in our analysis, partly because of the extra loading condition we have, and partly because of the lubrication theory approach we have used. Numerical techniques for problems of this type are discussed by, for example, Saxelby \& Aitchison (1986). However, we can expect the viscous jet satisfying (3.29) and (3.31) to relax on a dimensionless time scale of $O(1)$. If we take, as initial state, a uniform paste occupying $x>0, r<1$, then, if $R_{T} \sim O(1)$, conservation of mass must imply $w \sim 1$. This is inconsistent with $(3.2)_{3}$ unless $P$ changes rapidly near the free boundary $r=R$. This really suggests that the full equations (3.2) need to be reconsidered.

Our approximate viscous jet analysis successively had $\nabla \cdot u=0, \alpha=\alpha_{0}$, and then Stokes's equation. If these results were exact, then (3.2) 1,3 would imply $\nabla \cdot w=0$, and thus $\nabla \cdot\left(\mu_{1}^{-1} \nabla P\right)=0$. It is only the assumption that $\delta \rightarrow 0$ is a regular perturbation that allowed us to ignore $\alpha$ and $w$. However, the above 
Laplace-type equation for $P$ suggests that a further boundary condition for $P$ (or, originally, $p$ ) might be necessary. This is in line with the last sentence of Section 2 , where it is pointed out that one should have $w_{n}=0$ at the walls. The natural condition to suggest on $r=R$ for $p$ is (if $p$ is scaled like $P$ in (3.1))

$$
p=0 \text { on } r=R
$$

and possibly also (continuity of pressure at $x=0$ )

$$
p=\text { constant on } x=0 .
$$

We now have at least two possible choices. We have previously referred to the need for a constitutive relation relating $P$ and $p$. The simple choice $p_{\mathrm{c}}=P-p=$ constant is not exactly consistent with $\alpha=\alpha_{0}$. If we instead choose our constitutive relationship to be $\alpha=\alpha_{0}$, corresponding to an incompressible matrix, then we regain the previous jet approximation, with $p_{\mathrm{e}} \neq$ constant, and $p$ satisfies

$$
\begin{aligned}
& \nabla \cdot\left(\mu^{-1} \nabla p\right)=0, \\
& p=\text { constant }(>0) \text { on } x=0, \quad p=0 \text { on } r=R \text {, } \\
& \partial p / \partial n=0 \quad \text { on } r=1, \quad x>l_{s} / a \text {. }
\end{aligned}
$$

By use of the maximum principle, $p=0$ is the minimum interstitial pressure in the jet; however, this implies that the segregating flow is directed everywhere outwards from the jet boundary, which is inconsistent with the assumption of a steady solution, which requires $\int \mu^{-1}(\partial p / \partial n) d S=0$ along the jet boundary. In fact, this integral constraint together with $p=0$ (minimum) on the jet boundary requires also $\partial p / \partial n=0$ there, and the problem has no solution. Actually, differentiation of the interstitial sludge requires compaction of the coarse particulate, so that $\alpha=$ constant is unlikely to be a good model. A simple (static) assumption (cf. (2.4)) is

$$
p_{\mathrm{c}}=P-p=p_{\mathrm{c}}(\alpha) \quad\left(p_{\mathrm{c}}^{\prime}<0\right) .
$$

Simplification of (3.2) (with $p$ in $(3.2)_{3}$ ) then yields the equation for the sludge fraction $\alpha$ :

$$
\alpha_{\imath}+u \cdot \nabla \alpha=\delta(1-\alpha) \nabla \cdot\left(\mu^{-1} \alpha \nabla p\right) \text {. }
$$

For $\delta \neq 0,(4.6)$ is diffusive and we require $p$ (or $p_{\mathrm{c}}$ or $\alpha$ ) to be prescribed at the boundary. We have $\alpha=\alpha_{0}$ at $x=0$; thus $p=P-p_{\mathrm{c}}\left(\alpha_{0}\right) \geqslant 0$ there, while $p=0$ on $r=R$. The sense of extrusion is therefore out of the paste, as we expect. We can take $\alpha=1$ on $r=R$. It then follows from (4.6) that the viscous jet with $\alpha \approx \alpha_{0}$ is a valid approximation, except in a thin boundary layer of thickness $\sim\left(\delta \mu^{-1}\right)^{\frac{1}{2}}$, in which $\alpha$ jumps rapidly, $p$ drops sharply, and in which

Furthermore,

$$
|w-u| \sim\left(\delta \mu^{-1}\right)^{\frac{1}{2}}
$$

$$
\nabla \cdot u=\delta \nabla \cdot\left(\mu^{-1} \alpha \nabla p\right),
$$

so $u$ can be uniformly approximated in the jet by an incompressible flow field. However, it follows from (3.2), that, since $\nabla \cdot u \sim O(1)$ in the boundary layer, the total pressure $P$ has an $O(\beta \mu)$ jump across the layer, and thus the preceding 
viscous jet analysis must be adapted in this case to account for a nonzero normal stress near the boundary. This thickening boundary layer may be the partially segregated zone which is observed (see Fig. 1).

\section{Acknowledgements}

We wish to thank all those at the 1987 Study Group who contributed to the discussion, and particularly to John Lister and Grae Worster for their insights.

\section{REFERENCES}

Batchelor, J., Barry, J. P., \& Horsfall, F. 1973 Die swell in elastic and viscous fluids. Polymer 14, 297-99.

Bear, J. 1972 Dynamics of Fluids in Porous Media. New York: Elsevier.

Boulton, G. S., \& Hindmarsh, R. C. A. 1987 Sediment deformation beneath glaciers: Rheology and geological consequences. J. Geophys. Res. 92, 9059-82.

Clarke, G. K. S. 1987 Subglacial fill: A physical framework for its properties and processes. J. Geophys. Res. 92, 9023-36.

Covey, G. H., \& StANmore, B. R. 1980 Rheological behaviour of brown coal. Fuel 59, 123-27.

Davis, S. H. 1983 Contact-line problems in fluid mechanics. Trans. ASME, J. Appl. Mech. 50, 977-82.

Drew, D. A. 1983 Mathematical modelling of two-phase flow. Ann. Rev. Fluid Mech. 15, 261-91.

Hillel, D. 1980 Fundamentals of Soil Physics. London: Academic Press.

Middleman, S., \& Gavis, J. 1961 Expansion and contraction of capillary jets of Newtonian liquids. Phys. Fluids 4, 355-59.

SAKAI, M. 1979a Viscoelastic properties of a pitch and coke-pitch disperse system. Carbon 17, 139-44.

SAKAI, M. 1979b Dependence of viscoelastic properties of a carbon paste on grain size of coke. Carbon 17, 145-48.

SaXelby, C., \& Artchison, J. M. 1986 A numerical model of the glass sheet and fibre updraw processes. In: Industrial Numerical Analysis (ed. C. M. Elliott \& S. McKee), Oxford University Press. pp. 199-215.

Sørlie, M. 1984 Temperature stability of Søderberg anode pitch. Light Metals, Proc. Sess. AIME Annu. Meet., 113th, pp. 909-21.

TANner, R. I., LAM, H., \& BUSh, M. B. 1985 The separation of viscous jets. Phys. Fluids 28, 23-25.

Tørklep, K. 1988 Viscometry in paste production. Light Metals, Proc. Sess. AIME Annu. Meet., 117th, pp. 237-44. 